\title{
Monitoramento proativo e gerenciamento de rede da UFMT, usando a ferramenta Zabbix
}

\author{
Jonata B. M. dos Santos ${ }^{1}$, Jeison Gomes dos Santos ${ }^{1}$, Roberto B. de Oliveira Pereira ${ }^{2}$ \\ ${ }^{1}$ Secretaria de Tecnologia da Informação - Universidade Federal de Mato Grosso \\ (UFMT) \\ 78060-900 - Cuiabá - MT - Brasil \\ ${ }^{2}$ Instituto de Computação - Universidade Federal de Mato Grosso (UFMT) \\ 78060-900 - Cuiabá - MT - Brasil \\ \{jonata, jeison, robertobenedito\}@ufmt.br
}

\begin{abstract}
Resumo. Este artigo relata os benefícios alcançados por meio da implantação de uma solução de monitoramento proativo de rede na UFMT, usando a ferramenta Zabbix. ${ }^{1}$ A implantação foi realizada pelos técnicos da Secretaria de Tecnologia da Informação da UFMT e utilizou ambientes computacionais em container, por meio do uso da ferramenta Docker ${ }^{2}$. Foram definidos os requisitos funcionais essenciais para a implantação, sendo eles: envio de mensagem por aplicativo de celular, informando incidentes ocorridos na rede; e geração de relatórios e gráficos. Através da implantação, a Universidade obteve uma solução gratuita de monitoramento proativo de rede, possibilitando a eficácia e a eficiência das resoluções dos problemas, e diminuindo o tempo de resposta nos atendimentos de infraestrutura de rede.
\end{abstract}

Palavras-chave: Monitoramento, Redes, Zabbix.

\section{Introdução}

Com o passar dos anos, o uso de redes de computadores e da Internet nas organizações cresceu exponencialmente. Conforme pesquisa realizada pelo CETIC.br, no ano de 2017, $98 \%$ das empresas haviam utilizado a Internet nos últimos 12 meses. Além disso, $95 \%$ das empresas com até 49 funcionários já possuíam alguma infraestrutura em rede, sendo ela cabeada ou sem fio, bem como arquitetura intranet ou extranet [CETIC.br 2017].

Essa expansão, tanto de dispositivos como de equipamentos de rede, faz com que os serviços de tecnologia da informação que atuam nessas organizações e que dependem da rede para seu funcionamento, tenham um nível de disponibilidade maior, tornando evidente a necessidade de monitoramento [SILVA et al. 2015].

Para prover soluções inovadoras de Tecnologias da Informação e Comunicação (TIC's) para a comunidade da Universidade Federal de Mato Grosso (UFMT), a Secretaria de Tecnologia da Informação (STI) tem priorizado a profissionalização da gestão dos recursos de tecnologia da UFMT tendo, dentre outros, o compromisso com a eficiência de sua aplicação em benefício da administração e da comunidade, de forma socialmente integrada e comprometida [UFMT 2018].

\footnotetext{
${ }^{1}$ https://www.zabbix.com/

${ }^{2} \mathrm{https} / / / \mathrm{www} \cdot$ docker.com/
} 
Analisando o estado da arte com o foco em monitoramento e gerenciamento de rede, destaca-se o trabalho de [SILVA et al. 2015], em que se propõe a utilização da ferramenta Zabbix e do protocolo SNMP, não somente para monitoramento e controle, mas para resolução e antecipação de problemas relacionados a equipamentos de rede. $\mathrm{E}$ também, vale ressaltar o trabalho de [Mohr 2012], em que se avalia três ferramentas de monitoração de rede de código aberto, Zabbix, Nagios e Zenoss, sendo que o Zabbix mostrou ser a mais completa de todas, incluindo um bom poder computacional com uma interface bastante boa.

Baseado neste contexto, este artigo apresenta os resultados da implantação de uma solução proativa de monitoramento de ativos de rede da UFMT, utilizando o software Zabbix, tendo como propósito de melhorar a gestão dos recursos de TIC's, além de prover um nível maior de disponibilidade de serviços de rede à comunidade universitária, e aperfeiçoar o gerenciamento da rede lógica da instituição.

\section{Metodologias}

Para a implantação do software de monitoramento de rede Zabbix, foram definidas as etapas para execução, sendo elas: a geração da lista dos ativos de rede com os respectivos endereços IP's (Internet Protocol); a preparação do ambiente computacional; o cadastro dos ativos no sistema; o monitoramento dos ativos e análise dos gráficos.

$\mathrm{Na}$ etapa da geração da lista dos ativos de redes, foram elencados os dispositivos prioritários a serem monitorados, em que se destacam os $S_{w i t c h e s}{ }^{3}$ que disponibilizam as conexões de Internet para comunidade acadêmica. Inicialmente, encontrou-se dificuldade para obter o IP de cada Switch a ser monitorado, pois não havia nenhum local específico e centralizado que continha essa informação. Isso demandou o envolvimento de toda a equipe para unificar todas as informações numa única base de dados, com o intuito de obter o máximo dados dos ativos de redes possíveis. Por meio de buscas realizadas em planilhas documentais, a equipe obteve informações importantes dos dispositivos para 0 seu cadastro no sistema de monitoramento de rede, sendo eles: enderenços IP's, modelo, marca e entre outros.

Após realizada a etapa de levantamento de informações dos ativos de rede, o processo de implantação encaminhou-se para a etapa de preparação do ambiente computacional. Nesta etapa, foi feita a instalação e a configuração do Docker numa máquina virtual, bem como do Zabbix. Para que todo sistema funcionasse foi necessário a criação de 4 container no Docker, conforme a Figura 1, sendo 1 (um) para o Servidor Zabbix, 1 (um) para o Agente do Zabbix, 1 (um) para Servidor Web e 1 (um) para Banco de dados.

Ainda na Etapa de preparação do ambiente, foram feitas as configurações da comunicação do bancos de dados com o container do servidor Zabbix, as configurações de autenticação para acesso à interface web do Zabbix integrada com um Active Directory $(\mathrm{AD})^{4}$ e as configurações dos parâmetros de cada serviço e de cada container (Zabbixserver, Zabbix-Agent, Apache e PHP).

\footnotetext{
${ }^{3}$ Equipamento que possibilita a conexão de computadores em redes

${ }^{4}$ Serviço de rede da Microsoft que implementa o protocolo LDAP e que armazena informações sobre objetos em rede de computadores, tais como usuário, e disponibiliza essas informações a usuários e administradores desta rede.
} 

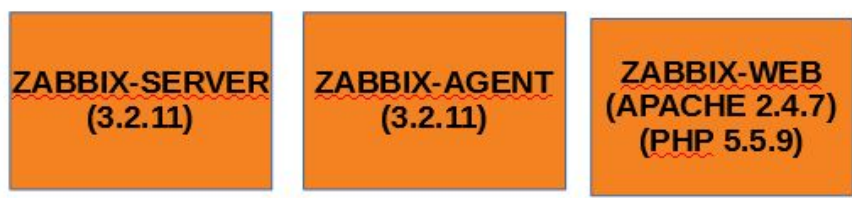

\section{DOCKER ENGINE}

\section{VM DEBIAN}

\section{VMWARE}

\section{HARDWARE}

Figura 1. Estrutura do ambiente do experimento

Em seguida, foram cadastrados os equipamentos de rede a serem monitorados no Zabbix. No cadastro, utilizou-se o protocolo $\mathrm{SNMP}^{5}$ e a função do Ping do protocolo ICMP ${ }^{6}$ para obter os status e os dados dos equipamentos em tempo real (uso da memóriaram, uso da CPU, taxa de tráfego de cada Interface). A Figura 2 expõe um exemplo de equipamentos de rede cadastrados.

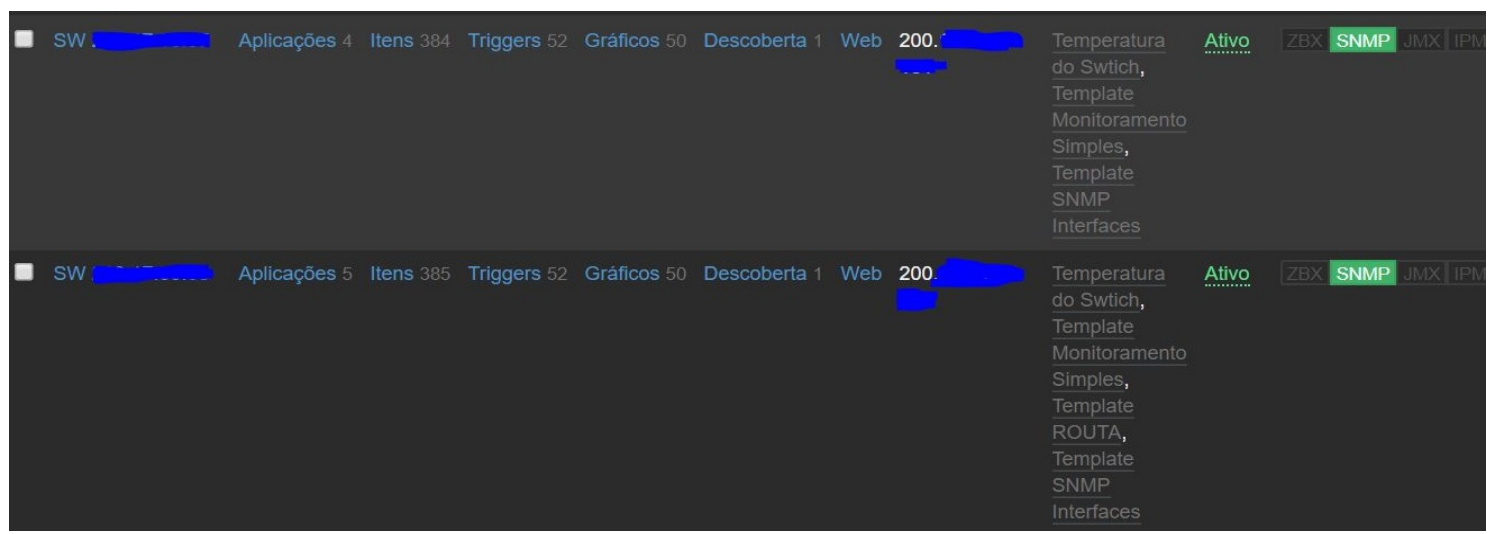

Figura 2. Exemplo de equipamentos cadastrados

Já na última etapa, foram criados os scripts para o encaminhamento de mensagem de notificação de incidente aos administradores de rede, no Telegram ${ }^{7}$. Os scripts desenvolvidos em Pyhton $^{8}$, tem a funcionalidade de notificar qualquer anomalia que possa ocorrer com os equipamentos de rede cadastrados. Além disso, foram analisados os gráficos gerados pelo sistema, tendo como finalidade de ter informações sobre os equipamentos cadastrados, e também, informações sobre a rede de modo geral, como por exemplo, o tráfego de rede.

\footnotetext{
${ }^{5}$ Protocolo para gerenciamento de dispositivos em redes IP

${ }^{6}$ Protocolo que possibilita detectar e relatar condições de erro em redes de computadores

${ }^{7}$ https://www.telegram.org/

${ }^{8}$ https://www.python.org/
} 


\section{Resultados}

Com a implantação do Zabbix para o monitoramento da rede da UFMT, a equipe de redes da STI obteve mais uma ferramenta para auxiliar nos atendimentos relacionados aos serviços de conectividade de rede. Graças ao uso do Zabbix integrado com o Telegram, que possibilita o envio de notificações de incidentes de rede da UFMT, em tempo real, a equipe conseguiu realizar atendimentos proativos, além de dimunir tempo de atendimento aos incidentes que ocorreram na rede da UFMT.

Conforme a Figura 3, foram encaminhadas à equipe técnica responsável pelo gerenciamento da rede, as notificações informando os incidentes que ocorreram com os equipamentos monitorados no Zabbix. A partir das notificações instantâneas, a equipe técnica realizou o atendimento proativo, diagnosticando o problema e aplicando solução definitiva, consequentemente, diminuindo o tempo de resposta para o atendimento.

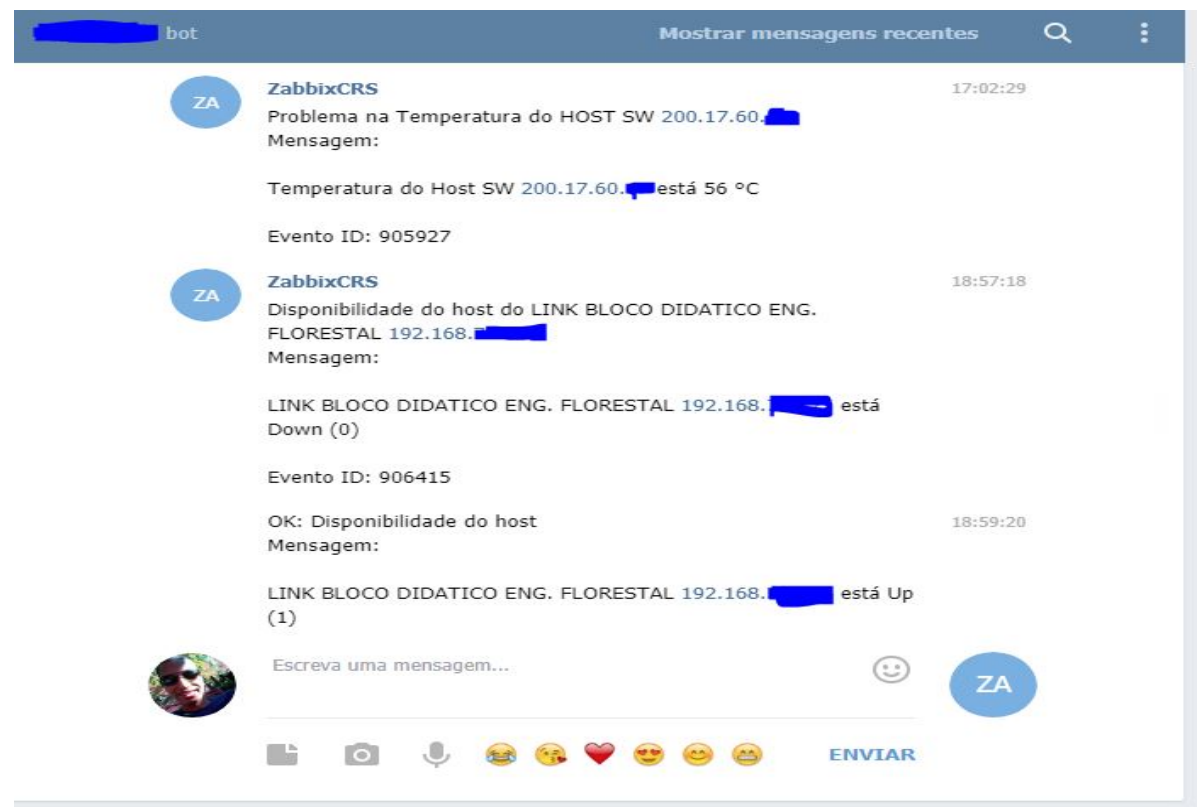

Figura 3. Alertas Recebidas no Telegram

Além das notificações de incidentes de rede, outro benefício importante adquirido com a implantação da ferramenta, foi a possibilidade de geração de gráficos e relatórios a partir dos dados capturados e armazenados.

A análise dos dados gerados através do monitoramento de rede possibilitou a verificação da disponibilidade do serviço de conectividade de rede em cada unidade acadêmica/administrativa da universidade. Como exemplo, verificou-se o serviço de conectividade oferecido para Gráfica Universitária, durante o período 21 de Julho de 2019 a 20 de Agosto de 2019, conforme a Figura 4.

Durante o período analisado, nota-se que no dia 09 de Agosto de 2019, o serviço de conectividade foi interrompido por completo. Associando o período do incidente registrado no Zabbix com a causa do incidente registrado no sistema de chamado, obtêm informações de suma importância para as tomadas de decisões, por exemplo: o setor que teve incidentes recorrentes, com a mesma causa, em certo período de tempo. 


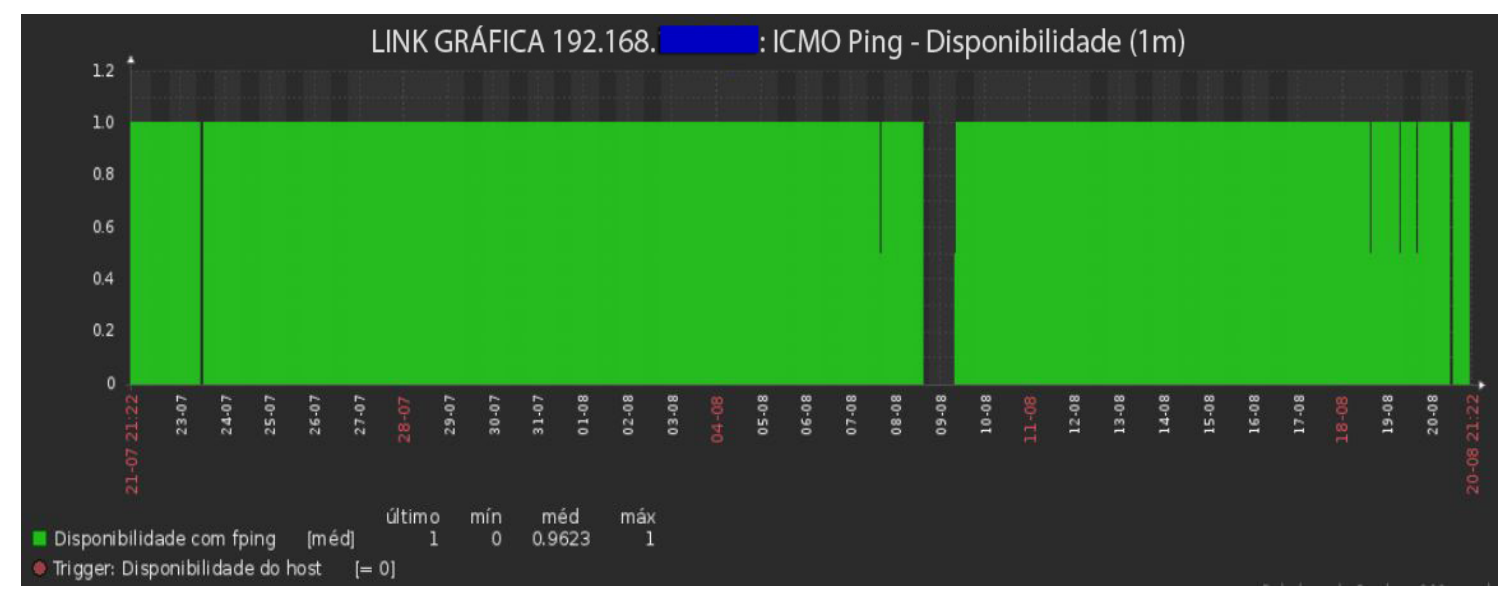

Figura 4. Gráfico de disponibilidade do LINK da Gráfica Universitária

Além disso, realizou-se análise relativa aos níveis de $\mathrm{SLA}^{9}$ (Service Level Agreement) do serviço de conectividade de rede das unidades da UFMT, de acordo com os dados apresentados na Figura 5. A numeração de cor verde representa o nível de SLA do serviço, em porcentagem. A numeração de cor vermelha representa o tempo de indisponibilidade do serviço, em porcentagem.

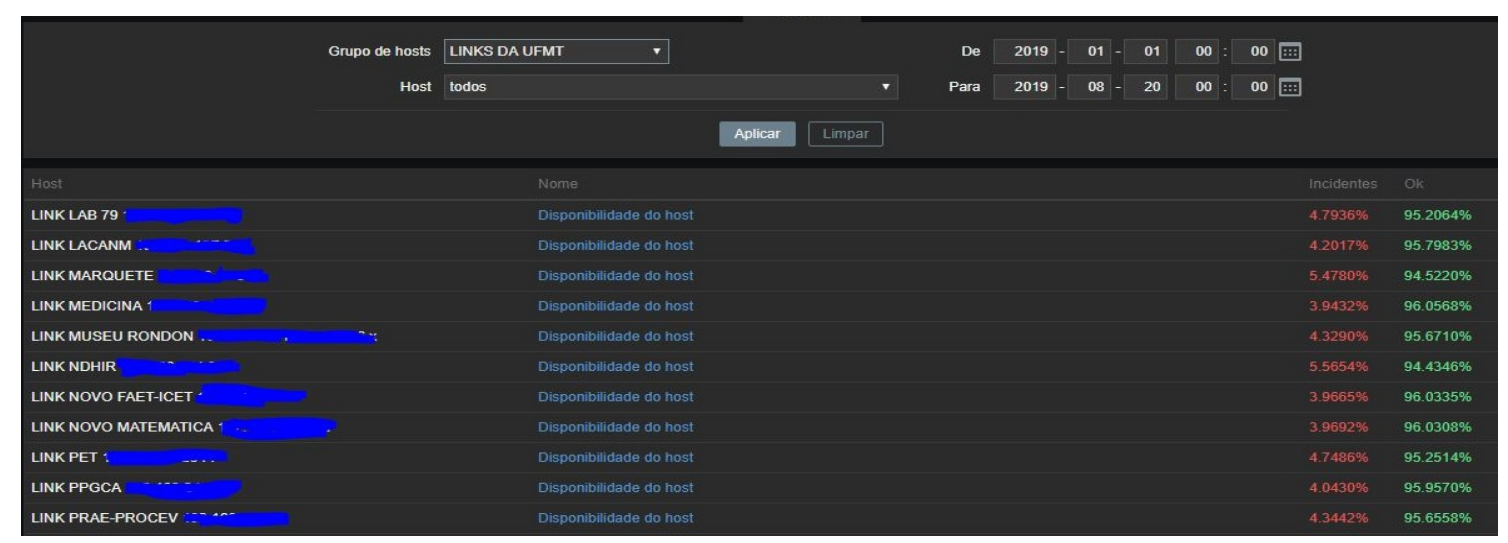

Figura 5. Relatório de disponibilidade de rede das Unidades

Por último, analisou-se o tráfego de rede em equipamentos cadastrados. A título de exemplo, averiguou-se o tráfego de rede, em $M B$ (MegaByte), na conexão que fornece o sinal de Internet para todos os setores da UFMT, conforme a Figura 6. Nota-se que no período das $14 \mathrm{~h}$ até $17 \mathrm{~h}$ do dia 16 de Agosto de 2019, teve tráfego médio de $120 \mathrm{MBs}$ de download e 166 MBs de upload, sendo que a taxa mais alta durante o período analisado foi de $322 \mathrm{MBs}$ de download e $404 \mathrm{MBs}$ de upload. Estas informações fornecem subsídios para que o gestor possa tomar decisões em relação a necessidade da escalabilidade da rede.

\footnotetext{
${ }^{9}$ Documento que representa o contrato de um fornecedor com o cliente, em que estabelece requisitos básicos para entrega de serviço de TI.
} 


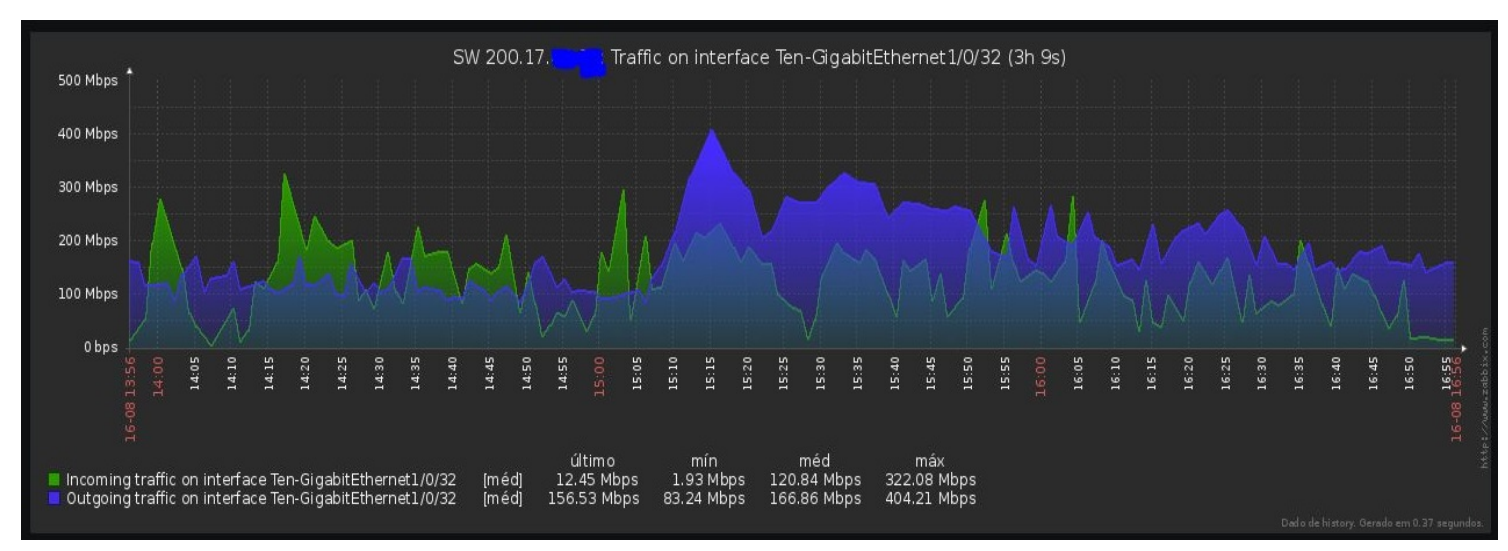

Figura 6. Gráfico de tráfego de conexão de rede do SW

\section{Conclusões}

Os resultados apresentados neste trabalho confirmam a viabilidade do uso Zabbix, juntamente com o Telegram, como uma solução de monitoramento proativo de rede. Com a implantação, a equipe pode gerenciar e monitorar, de forma eficaz e eficiente, os ativos de rede da Universidade, disponibilizando um melhor serviço de conectividade à Internet aos usuários finais.

Com a consolidação da Implementação da solução de monitoramento de rede Zabbix na UFMT, pode-se observar muitos benefícios a Gerência de Manutenção de Rede Lógica (GMRL) da UFMT. A ferramenta ajudou a reduzir significativamente o tempo de resposta para atendimento aos usuários, além de que, o monitoramento de rede ajudou a melhorar as tarefas dos técnicos de rede, facilitando a detecção da falha dos serviços de rede ou até mesmo a sua prevenção, ajudando a reduzir consideravelmente o downtime causado por problemas rotineiros.

Trabalhos futuros poderão realizar a integração do Zabbix com as ferramentas Grafana $^{10}$ e GLPI ${ }^{11}$. E também, os serviços de redes a serem monitoradas poderão ser ampliados, dando ênfase aos hosts do Datacenter e câmeras de segurança.

\section{Referências}

CETIC.br (2017). TIC Empresas - $2017 . \quad$ Disponível em: https://www.cetic.br/pesquisa/empresas/indicadores. Acesso em: 26 ago. 2019.

Mohr, R. F. (2012). Análise de ferramentas de monitoração de código aberto. Monografia (Bacharel em ciência da Computação), UFRGS (Universidade Federal do Rio Grande do Sul), Porto Alegre, Brasil.

SILVA, W. M. C., M., M. R., and MARTINS, R. S. (2015). Análise e Gerenciamento de Redes Usando Metodologia Proativa com Zabbix. HOLOS, 8:277-289.

UFMT (2018). Relatório de gestão 2016 - 2018 : governança, qualidade acadêmica e pluralidade. Editora da Universidade Federal de Mato Grosso. Disponível em: http://editora.ufmt.br/download/2019/Relat\%C3\%B3rio\%20de\%20Gest\%C3\%A3o\% 202016\%20-\%202018\%20-\%20eBook.pdf. Acesso em: 27 ago. 2019.

\footnotetext{
${ }^{10} \mathrm{https}: / /$ www.grafana.com

${ }^{11} \mathrm{https}: / /$ www.glpi-project.org/pt-br/
} 